\title{
RESEARCH ON THE PROTOTYPE OF RAIL CLEARANCE MEASUREMENT SYSTEM
}

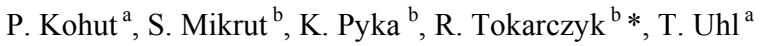 \\ a AGH University of Science and Technology, Dept. of Mechatronics and Robotics, 30-059 Cracow, Poland \\ - (pko, tuhl)@agh.edu.pl \\ ${ }^{\mathrm{b}}$ AGH University of Science and Technology, Dept. of Geoinformation, Photogrammetry and Environmental Remote \\ Sensing, , 30-059 Cracow, Poland - (smikrut, krisfoto, tokarcz)@agh.edu.pl
}

Commission IV WG IV/2, IV/4

KEY WORDS: rail clearance, measurement system, photogrammetry, laser scanning, integration,

\begin{abstract}
:
Measuring of railway structure clearances is a very practical issue, and the operation itself is performed all over the world using various measuring systems. The measurement is aimed chiefly at determining the limits of space that no structure (e.g. a building) located close to the railway track may cross. The paper demonstrates flaws and advantages of particular systems and provides a technological summary of measuring equipment utilized in them. In those systems the measurement of structures is referred to the global system of coordinates, and later, following the detection of rail heads, a transformation to the rail axis arrangement takes place. The preliminary analysis leads to a conclusion that the accuracy of rail clearance measurement is higher in the case of specialized systems as compared to universal ones. On the basis of experience drawn from literature and earlier tests, a decision has been made to proceed with the construction of a rail clearance measurement system in Poland, at the University of Science and Technology in Cracow. This paper presents a research on the prototype of the rail clearance measurement system constructed at the University. The heart of the system involves laser scanners installed on a flatcar and digital cameras. The base of the system includes scanners, which capture data in a continuous way, and photogrammetric cameras, which yield digital images.

The cloud of points obtained from a laser scanning is colorized, which causes that such information can replace a classical texture model.
\end{abstract}

\section{INTRODUCTION}

\subsection{Goal}

Measuring of railway structure clearances is a practical issue, performed all over the world, using various measuring systems. The measurement is aimed chiefly at demonstrating the limits of space that no structure (e.g. a building) may cross. Clearance measurements can be performed either statically, or dynamically in the course of the measuring system's travel on a railway track.

The authors of this paper are presently implementing a project, and one of its tasks is developing a prototype of such system. The project is being carried out by research team from the AGH University of Science and Technology, upon the commission of Polish Railways. The main goal of the project includes research on a prototype of a system of rail clearance measurement and railway codification. In this paper, the authors focus on the first part of the project, i.e. developing of a prototype of a measuring system. To attain that goal, the authors have reviewed the existing systems (Section 1.2), and next, selected two solutions, which were examined, and for which test measurements described in Section 3 were performed.

Based on experiments, which had been conducted, the authors proposed a prototype of measuring flatcar to be tested in subsequent stages of the project (Section 4).

\subsection{Examples of Railway Clearance Measuring Systems}

The project authors have reviewed the existing measuring systems used to determine structure clearances, systematizing them from the simplest ones (e.g. LaserTEC electronic rail gauge) to the most modern and most technologically advanced mobile systems.

Based on their functionality, those systems were divided into:

- those dedicated to railway-specific applications, e.g. British Balfour Beatty Rail Technologies (BBRT,2011), German LIMEZ I, II, and III (Schewe et al., 1999; Meier, 2009) and Zoller + Fröhlich Profiler (Zoller+Fröhlich, 2010),

- two-way systems (e.g. Italian L-KOPIA / LKO B1 Clearance Laser System (L-KOPIA, 2012),

- universal mobile systems, with an option of rail clearance measuring, e.g. Austrian RIEGL system (Riegl, 2011) and German 3D Mapping Solution.

Systems currently in use are based mainly on three groups of methods:

a) the photogrammetric method,

b) the method of light profiles, which are applied by the laser light and recorded by means of a high-speed digital camera,

c) the method based on laser or radar scanner measurements.

Those most advanced systems combine the above-referenced measurement methods. Within the research framework, the systems were reviewed, and features of measuring devices were summarized as regards their flaws and advantages, so that the proposed prototype could be as much optimal, as possible. On the basis of those research works, the authors have worked out

\footnotetext{
* Corresponding author.
} 
assumptions for a measuring system responding to the questions about selection and configuration of measuring equipment (photogrammetric cameras, laser scanners, GPS/INS equipment, etc.).

\section{ASSUMPTIONS OF A MEASURING SYSTEM}

\subsection{Assumptions of a Measuring System}

When developing the assumptions, a rule was established that the system would be installed on a flatcar, hauled by a railway engine with the maximum speed of $100 \mathrm{~km} / \mathrm{h}$.

Due to the nature of equipment tested, it was decided that measuring systems should be placed in the back part of the railway engine. Such solution offers the following advantages:

- it is possible to utilize a standard railway flatcar,

- data control and recording systems, a scanner system, a georeferential system and a photogrammetric system can be installed on the flatcar, applying variant modes of their mutual arrangement,

- scanning made diagonally in relation to the rail track axis can be performed in almost full $360^{\circ}$ profiles (in the event that the scanning-photogrammetric system is installed before the railway engine, diagonal scanning is performed in $0-180^{\circ}$ profiles).

Once the detailed review of existing systems had been completed, the authors decided that the system should include the following components:

- a set of 2D scanners profiling in three directions: perpendicularly to the rail track axis and in two diagonal directions,

- a set of four cameras to colorize the cloud of points originating from laser scanning,

- a GNSS/IMU geo-referential module integrated with scanners and cameras,

- a photogrammetric module composed of two stereovision systems of canonical or convergent geometry.

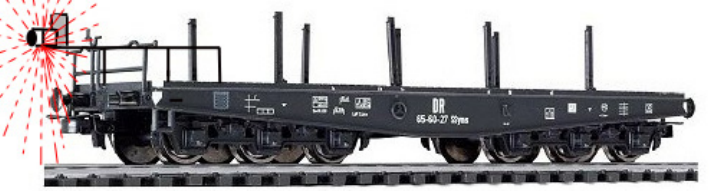

Figure 1. Schematic diagram of flatcar with a single profiling scanner

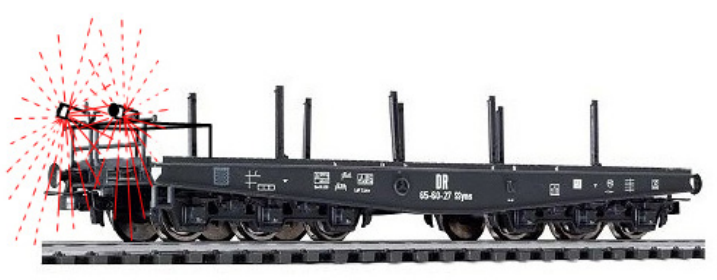

Figure 2. Schematic diagram of flatcar with two diagonal scanners
In order to compare potential and measuring accuracy offered by various systems, the authors decided to carry out separate measurements using two independent methods:

System I (based on a phase scanner):

- a scanner profiling perpendicularly to the direction of travel,

System II (based on pulse scanners):

- scanners profiling in two diagonal directions and integrated with photogrammetric cameras.

Schematic diagrams of both systems are shown in Figs. 1 and 2. Fig. 1 shows flatcar with System I, whereas Fig. 2 depicts the idea of System II (installation of two diagonal scanners).

\subsection{Description of used measurement systems}

System I was to test the technology of measurement by means of the so-called "railway dedicated" device. A Z+F PROFILER 9000 profiling scanner, coupled with an odometer, was applied. That system (Z+F PROFILER 9000 with an odometer) operates in the mode of data acquisition being performed perpendicularly to the train travel direction and conducts measurement of data within the track axis system. To acquire data, Z+F PROFILER 9000 utilizes only a single scanner, which is capable of acquiring them in $360^{\circ}$ profiles, as opposed to the earlier model - PROFILER 6007 DUO - which employed two scanners.

R088ND3 odometer by Wenglor was installed for the period of measurements. For safety reasons, Z+F PROFILER 9000 was put on a flatcar (car type RES), on a previously prepared supporting structure (Fig . 3).

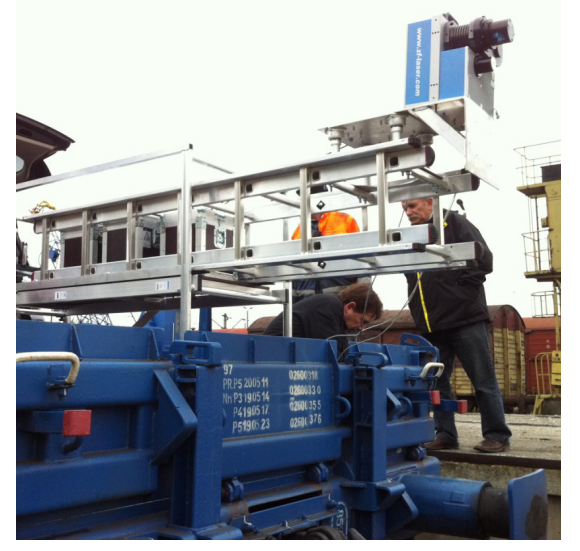

Figure 3. System I - Profiler 9000 scanner during installation

The Z+F PROFILER 9000 measuring system is the fastest scanner that provides data in the form of two-dimensional profiles. This is a phase scanner, allowing to record more than 1 million of points per second, with the maximum recording speed of 200 profiles per second. Thanks to those parameters, it is possible to obtain very small distances between sections, even at high speeds. The second measuring system (System II Fig. 4) was based on a RIEGL solution, namely VMX-250.

During research, the equipment configuration has been modified and extended by additional NIKON 7000 cameras. The mobile RIEGL VMX - 250 system is a set of two Riegl VQ-250 scanners with a GPS unit, installed under an aerodynamic cover. The whole measuring system includes four digital cameras, whose viewing ranges can be determined individually for each camera, depending on project needs. 


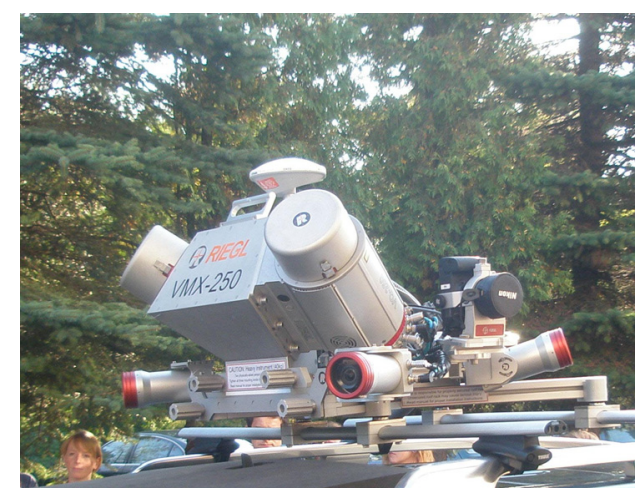

Figure 4. System II based on a Riegl solution (VMX 250).

The whole system was put on a truck and, together with the flatcar, participated in the measurement.

\section{TEST MEASUREMENTS}

\subsection{Selection of the Route}

For the performance of tests measurement, 3 railway track sections on the Warsaw-Cracow route were selected, each 10 $\mathrm{km}$ long, with different land features and elements so that to take into account the following:

- selected clearance infrastructure elements, such as bridges, viaducts, various type semaphores, traction poles, platforms, platform umbrella roofs, and a tunnel,

- a relatively diversified track geometry, including circular arcs and transition curves,

- a previously set geodetic control network with already monumented points. The latter was of a high significance since it considerably reduced project preparation time and costs.

A reference field geodetic measurement was performed to verify the accuracy of selected methods.

On the basis of measured geodetic control network, a measurement was conducted, which included the performing of profiles perpendicular to the rail direction and a tachymetric measurement of selected clearance infrastructure elements. Fig. 5 shows an example of track reference measurement with the use of the most recent LaserTEC device by GRAW.

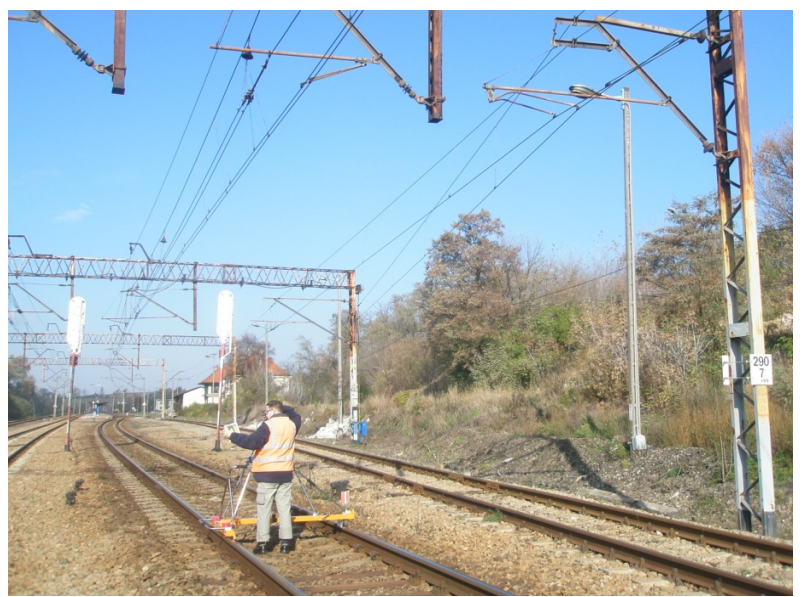

Figure 5. Track reference measurement with the use of the most recent LaserTEC device by GRAW

\subsection{Examination of Acquired Data Quality}

Following the data recording by means of the measuring flatcar (System I and II) and geo-referential measurements, has been proceeded with the examination of geometrical quality of acquired data.

In the first turn, conformity of the cloud of points obtained with the use of System II was examined based on control points. In the course of the measuring flatcar's travel, there were posts put along the track and their coordinates measured by GPS technique (precisely the post starting points). 16 control points were set along the travel route.

Next there were accuracy measurements on particular sections performed on the basis of selected sections, which were measured geodetically and with the use of a precision rail gauge (LaserTEC), with a millimetre accuracy.

Comparisons were made with reference to about 30 sections (involving geodetic measurements and in-house works in relation to the cloud of points - see example, Fig. 6). Deviations were measured in the section plane perpendicular to the track axis. The width of the section was selected depending on processed elements and ranged from 0.10 to $1.00 \mathrm{~m}$.

First measurements were performed on the System II cloud of points. Clearance was measured in 10 sections on the route near the village of Podleśna Wola, and in 21 sections in the vicinity of Słomniki station. 14 measurements were performed towards track 2 axis, while 17 measurements were performed towards track 1 axis. Deviations were measured in the section plane under '2000' geodetic coordinate system, while vectors from the cloud of points to reference points from geodetic measurements. Deviations were within the acceptable limit assumed to be $2 \mathrm{~cm}$.

Additionally, a measurement was performed near the railway station, of 32 points on the track and the already referenced 16 posts placed before the travel of the scanner. Measurement of deviations of posts and points was performed only for System II.

In addition to examining the geometric quality of the cloud of points, also the quality of data was examined as regards their preparation for further processing.

Within the framework of the task, an attempt at an automatic classification of the whole of the cloud of points was made, and additionally selected elements were classified manually in those track sectors where geodetic measurements had been made, i.e. in the tunnel, before the tunnel, and at the railway station.

With reference to selected places, a comparison of sections obtained by means of both Systems was performed. Exemplary comparison results are shown in Figs. 7 and 8.

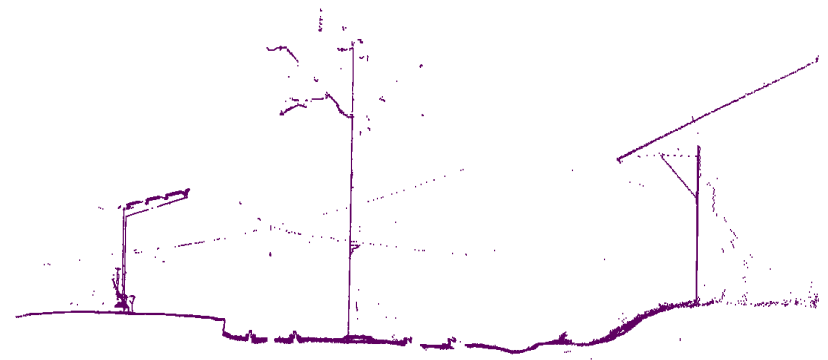

Figure 6. Cross section through the railway station at Słomniki, obtained with System II 
It is worth noting that systems of decidedly different reference bases were tested. One system referred measurements only to the track, while the other located them within a global system. Both solutions have their flaws and advantages. Measurements referred to tracks are less complicated, do not require GNSS/IMU modules and yield higher clearance measurement accuracy rates. Global measurements enable not only clearance measurements but also examinations of some of track geometry parameters (straight line sections, transition curves, circular arcs, superelevations). The access to an absolute orientation is at the expense of a slightly lower accuracy, which places itself close to the acceptable limit, or exceeds it a little.

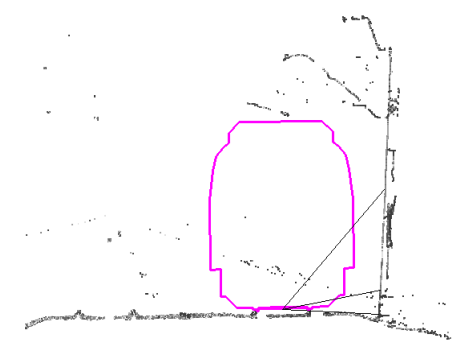

Figure 7. Clearance with measured railway infrastructure elements obtained from rail gauge measurements, at the background of the cloud of points from the Z+F Profiler 9000 system

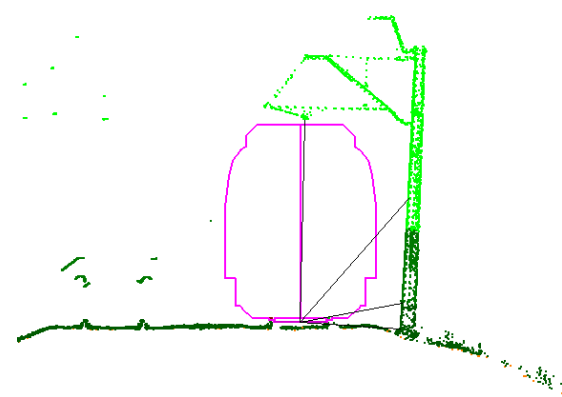

Figure 8. Clearance with measured railway infrastructure elements obtained from rail gauge measurements, at the background of the cloud of points originating from the Riegl VMX - 250 system

\section{DEVELOPMENT OF MEASUREMENT FLATCAR MODEL PROTOTYPE}

\subsection{Conclusions from Field Measurements}

Following the data analysis and examination of their quality, a summary was prepared aiming at the indication of flaws and advantages of particular systems. It was concluded that the best solution would be a hybrid model, which combines both solutions. In fact, System II could not cope well with recording small elements, which means there were gaps in data covering, e.g. roadside indicators. Here it seems to be appropriate to reinforce the cloud of points with a third scanner, set perpendicularly to the travelling direction.

In System I there was a quite high level of data noise. Without support from the cloud of points from System I it was often difficult to unambiguously identify objects.
As regards image recording and arrangement of cameras, after having analysed the recorded by Nikon cameras material, the authors came to a conclusion that it was the forward view, which was more useful than side view, and that conclusion would be taken into account when developing the prototype.

\subsection{Proposal of Measurement Flatcar Prototype}

Conclusions drawn from field measurements and development of a preliminary concept led to defining the measurement system prototype model. It has been acknowledged that the system should incorporate the following units:

- a scanner module,

- an image module,

- a GNSS/IMU recording module,

- a module for precision recording of rail heads.

The scanner module should include minimum three 2D scanning lasers. Two pulse-type scanners may be set diagonally and configured to perform measurements within the range of 150 metres, with a minimum frequency of $100 \mathrm{~Hz}$ for each scanner. The perpendicularly profiling scanner may be a phase type of a frequency of $300 \mathrm{~Hz}$. Presently, the maximum profiling frequency for scanners available on the market is 200 $\mathrm{Hz}$, therefore one may consider a temporary application of two scanners. The system of scanners should provide a cloud of points with the average distance between perpendicular profiles of not more than $10 \mathrm{~cm}$ at the travelling speed of $100 \mathrm{~km} / \mathrm{h}$ (with the density of points in profile planes ranging from several to a dozen or so millimetres). This will ensure detection of elements in the clearance area and in its close surroundings of the minimum dimensions of $10 \times 10 \mathrm{~cm}$. If two scanners are applied, of profiling frequency of $200 \mathrm{~Hz}$ each, the resolution of detail detection will increase, reaching the level of $7 \mathrm{~cm}$ in the direction parallel to the track axis. There are no technological barriers to increasing resolution in the perpendicular direction.

The laser scanners must be rigidly integrated with the GNSS/IMU module. Another element is a set of 4-8 high resolution photogrammetric cameras. Two pairs of cameras constitute a set of photogrammetric cameras and are used for performing additional, supplementary measurements.

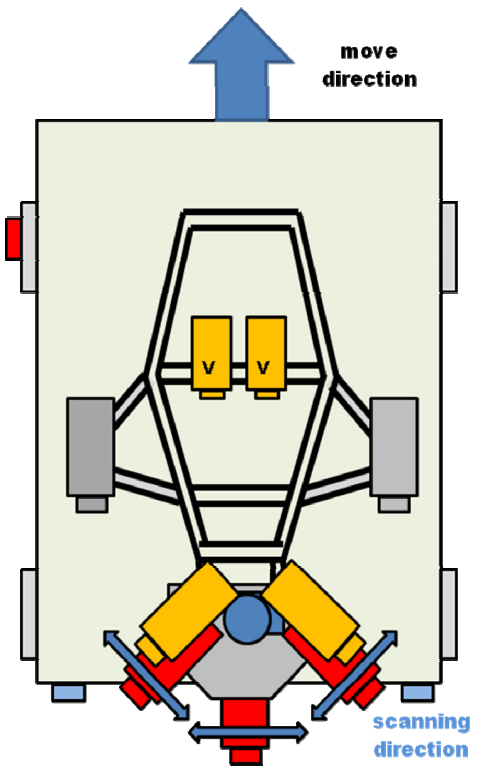

Figure 9. Model of measuring flatcar 
They can also be used jointly with remaining cameras to colorize the cloud of points. In order to verify travel trajectory and to release cameras, an odometer is fixed to one wheel of the axle set. The whole equipment set is mounted on a structural section fixed to the flat car deck. The diagram of arrangement of particular system elements in different variants of installation of high-resolution digital cameras is shown in Fig. 9. The module of 3 scanners is marked in red, 4 photogrammetric cameras are marked in grey and yellow ( 2 black and white cameras and 2 colour cameras, respectively), and 2 colour video cameras are marked in yellow with additional " $v$ " letters on them. Red rectangle on the wheel shows the position of odometer.

\section{SUMMARY AND CONCLUSIONS}

The goal of the paper was to show results of research works completed by a research and development team from the AGH University of Science and Technology in Cracow, Poland, under the project of constructing a prototype of flatcar to measure railway clearances.

In the first part of the project a review of existing systems was made (Section 1.2). Based on preliminary literature survey, two different measurement systems were selected to test flaws and advantages of particular methods. Next test measurements, described in Section 3, were performed on a selected railway route sector.

The final stage of research was proposing a model of a groundbased scanner railway lorry with a geo-referential module and a photogrammetric module, which was described in Section 4.

It was assumed that using presently available scanners and upon the application of perpendicular scanning together with diagonal one, it would be possible to guarantee detection of all obstacles in the track-side area, with one of their dimensions higher than $5 \mathrm{~cm}$. It is, however, impossible to fully identify what a reflecting surface is. Therefore, upon detection of so small, "single-point details" it is necessary to use cameras and to look over images showing the place under discussion. Experience demonstrates that looking over images was the weakest side of the photogrammetric technology due to the labour intensity and possibility of omitting objects that enter the clearance area. Also measurement performed on a 3D stereomodel is manual, with a low rate of automation. This results from the specificity of the railway zone space, in which objects subjected to measurement are relatively small, often lofty, with a relatively distant background recorded between them, which radically changes the stereoscopic model depth. Hence photogrammetric support in the form it is used now should be reduced to an absolute minimum - to clarify doubts that appear during measurements in the cloud of points (that is, incidentally).

One of conclusions originating from the completed research works is a statement that the cloud of points should develop from profiling in different directions: perpendicularly and diagonally to the track axis. Measurement performed on a cloud of points obtained from perpendicular profiling gives better accuracy rates but it not always is capable of detecting the existence of objects, especially those small ones, along the railway track and extending crosswise. Scanning performed in different directions increases the density of the cloud of points, which density is of critical importance for the identification of details. Average distance between profiles in tested clouds of points varied from 0.05 to $0.20 \mathrm{~m}$, with the smaller interval reached only at low travelling speed. Systems used during tests were not able to provide a sufficient data density for a travel with the speed of $80 \mathrm{~km} / \mathrm{h}$. A specific sufficient density equals the possibility of detecting details of several centimetres in size. Therefore, the target system, which has to operate at the speed of $100 \mathrm{~km} / \mathrm{h}$, must utilize both different directions of profiling, and scanners of higher performance rates.

\section{REFERENCES}

BBRT 2011, Balfour Beatty Rail Technologies materials for the AGH University of Science and Technology. "Limited Gauging Capability Overview and Consideration of Areas of Support in PKP PLK's Gauging Research Project".

Schewe H., Holl J., Gründig L., 1999. LIMEZ Photogrammetric Measurement of Railroad Clearance Obstacles. Third Turkish-German Joint Geodetic Days, Istanbul/Turkey. Towards a Digital Age, Volume II, page 721727.

Meier J., 2009, a presentation titled LIMEZ III - Der neue Lichtraummesszug der Deutschen Bahn "Railborn High-Speed Laserscanning". 3. Hamburger Anwenderforum Terrestrisches Laser-Scanning. Hamburg.

http://www.geomatikhamburg.de/tls/tls2009/images/14_tls2009 _meier.pdf (15 Apr. 2012)

L-KOPIA, 2012.

http://www.lko.com/ (15 Apr. 2012)

Riegl, 2011.

http://www.riegl.com/uploads/tx_pxpriegldownloads/10_DataS heet_VMX-250_26-09-2011.pdf(15 Apr. 2012)

Zoller+Fröhlich, 2010.

http://pdf.directindustry.com/pdf/zoller-frohlich/brochure-z-fprofiler-6007-duo/66967-187670.html (15 Apr. 2012)

\section{ACKNOWLEDGEMENTS}

This work was funded by AGH University of Science and Technology, project No: 11.11.150.949 and 5.72.130.151. 